\title{
The impact of inorganic nitrate consumption on endothelial function measured by flow-mediated dilatation: a systematic review of randomised controlled trials
}

\author{
B. Celik ${ }^{1}$, E. N. Muriuki ${ }^{1}$, G.G.C. Kuhnle ${ }^{1}$, J.P.E. Spencer ${ }^{1}$ and C.E. Mills ${ }^{1}$ \\ ${ }^{1}$ Hugh Sinclair Unit of Human Nutrition, University of Reading, Whiteknights, Reading, UK
}

Inorganic nitrate found in beetroot and green leafy vegetables has been demonstrated to improve cardiovascular disease risk factors by increasing circulating nitric oxide (a key vasodilator), via the nitrate-nitrite-NO pathway ${ }^{(1)}$. Inorganic nitrate supplementation is associated with lower blood pressure ${ }^{(2)}$, an effect that may be mediated by an improvement of endothelial function. In this systematic review, we aimed to investigate the impact of inorganic nitrate on endothelial function (an independent risk factor for cardiovascular disease) in adults.

A systematic review of randomised controlled trials (RCTs) assessing the impact of inorganic nitrate on endothelial function was performed. PubMed, Web of Science, and Scopus were used for the literature search and studies published from inception to July 2020 were included. The search was conducted with following pre-defined search terms: "nitrate" OR "nitrite" OR "nitric oxide" OR "Beetroot" OR "beet root" AND "flow-mediated dilation" OR "dilation" OR "vasodilation" OR "FMD" OR "endotheli" $A N D$ "human" AND "randomized controlled trial*". Inclusion criteria were RCTs testing the oral administration of inorganic nitrate as dietary or non-dietary forms versus a control, using adult males and female subjects regardless of health status, age or ethnicity, measuring endothelial function by flow-mediated dilatation (FMD; the gold standard measure). Papers for inclusion were reviewed by two independent assessors. The Jadad risk of bias score was used to assess risk and quality of each study ${ }^{(3)}$.

In total, 3,521 studies were identified after the electronic search. Once all duplicates were eliminated $(n=465)$, abstracts and titles of the remaining 3,056 articles were screened. Of these $n=3,032$ were removed and the remaining 24 studies were reviewed in full, this led to 4 further studies being excluded. A total of 20 studies remained; all were included in the review. Twelve studies were acute and 8 studies were chronic. In total, 11 studies were completed among healthy subjects. Non-dietary inorganic nitrate (sodium and potassium nitrate) intake ranged ( $\mathrm{n}=5$ studies) from $\sim 6$ to $24 \mathrm{mmol}$. All other studies used beetroot juice; volumes ranged from 140 to 250 $\mathrm{mL}$. Beneficial effects on endothelial function were seen in $80 \%$ of the studies (FMD $\%$ changes from $0.1 \%(95 \% \mathrm{CI}-0.4$ to 0.4 ) to $1.5 \%$ ( $95 \%$ CI 0.4 to 2.7 ) were observed); results from the remaining $20 \%$ were neutral. Interestingly, all acute studies that tested participants with pre-existing medical conditions, found a positive impact on endothelial function. From the risk and quality assessment, all studies were classified as a good quality.

Inorganic nitrate supplementation seems to be related to improved endothelial function in both acute and chronic studies. This improvement in an independent cardiovascular disease risk marker could contribute towards cardiovascular disease prevention. Future meta-analysis will test statistical significance of these results. These findings should be supported by more chronic and welldesigned studies.

\section{References}

1. Webb AJ, Patel N, Loukogeorgakis S, et al. (2008) Hypertension 51, 784-790.

2. Siervo M, Lara J, Ogbonmwan I, et al. (2013) J Nutr 143(6), 818-826.

3. Jadad AR, Moore RA, Carroll D, et al. (1996) Control Clin Trials 17(1), 1-12. 